\title{
Surface Realization with the Intersection Segment Functional
}

\author{
Stefan Hougardy, Frank H. Lutz†, and Mariano Zelke*
}

\begin{abstract}
Deciding realizability of a given polyhedral map on a (compact, connected) surface belongs to the hard problems in discrete geometry, from the theoretical, the algorithmic, and the practical point of view.

In this paper, we present a heuristic algorithm for the realization of simplicial maps, based on the intersection segment functional. The heuristic was used to find geometric realizations in $\mathbb{R}^{3}$ for all vertex-minimal triangulations of the orientable surfaces of genus $g=3$ and $g=4$. Moreover, for the first time, examples of simplicial polyhedra in $\mathbb{R}^{3}$ of genus 5 with 12 vertices were obtained.
\end{abstract}

\section{Introduction}

A polyhedral map on a surface is a (finite) set of polygons (with at least three sides), which are glued together (topologically) along edges to form the surface, such that there are no self-identifications on the boundaries of the polygons, and two polygons are either disjoint or intersect in exactly one edge or one vertex only. We thus can think of a polyhedral map as a combinatorial model for a surface.

For a given polyhedral map it is natural to try to visualize it as a polyhedron in threespace or in higherdimensional space $\mathbb{R}^{d}$, such that every polygon is the convex hull of its vertices and two polygons are either disjoint in $\mathbb{R}^{d}$, they intersect in a common edge and are not coplanar, or they intersect in a common vertex only. Such a realization usually is called a geometric or polyhedral realization, with straight edges, plane polygons, and no non-trivial intersections (with neighboring polygons being not coplanar).

Example 1: A polyhedral map on the 2-sphere $S^{2}$ consisting of the polygons 123, 12478, $13568,2354,4567$, and 678 together with a corresponding realization in $\mathbb{R}^{3}$ is displayed in Figure 1.

Realizability of maps on the 2-sphere $S^{2}$ was proved by Steinitz ([57], [58]; cf. also [30, Ch. 13], [69, Lec. 4]): Every polyhedral map on the 2-sphere $S^{2}$ is geometrically realizable in $\mathbb{R}^{3}$ as the boundary complex of a convex 3-polytope.

However, not all polyhedral maps are realizable. For example, simple polyhedral maps (i.e., maps with all vertices of valence three) on surfaces different from the 2 -sphere $S^{2}$ are not realizable in any $\mathbb{R}^{d}$ (see Grünbaum [30, Ex. 11.1.7, Ex. 13.2.3]).

\footnotetext{
*Supported by the DFG Research Center MatheON "Mathematics for Key Technologies", Berlin

†Supported by the DFG Research Group "Polyhedral Surfaces", Berlin
} 

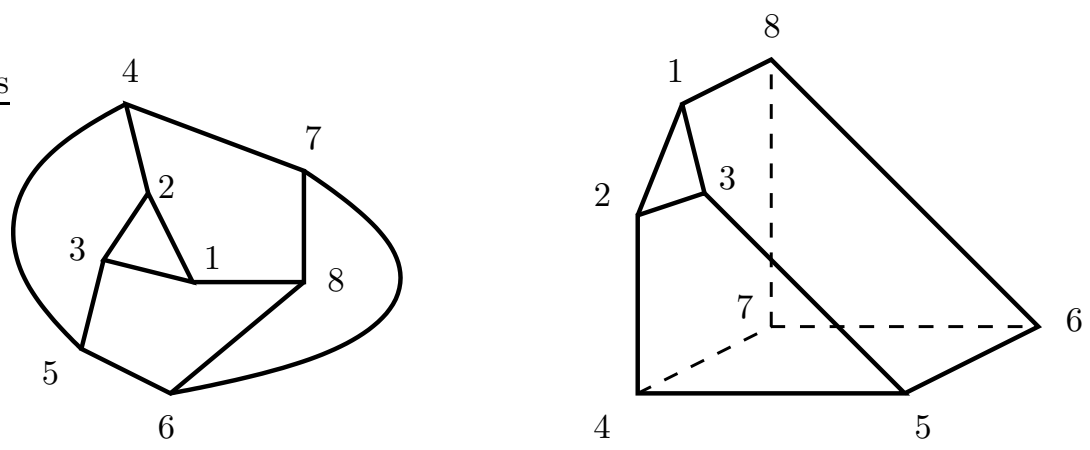

Figure 1: A polyhedral map on $S^{2}$ and a corresponding geometric realization in $\mathbb{R}^{3}$.

Example 2: All 6-3-equivelar maps on the torus (i.e., maps consisting of only 6-gons with every vertex belonging to exactly three 6 -gons) are simple and therefore cannot be realized in any $\mathbb{R}^{d}$. The smallest example (see Figure 2) of the family is the combinatorial dual of Möbius' 7-vertex triangulation of the torus [44]. A "realization" of this 6-3-torus with flat, but non-convex 6-gons was given by Szilassi [64], the Szilassi-torus.

Betke and Gritzmann [9] found a further combinatorial obstruction to geometric realizability: Let $W$ be any subset of the set of odd valent vertices of a polyhedral map $M^{2}$ and let $F_{W}$ be the set of facets containing some vertex of $W$. If $2\left|F_{W}\right| \leq|W|$, then $M^{2}$ is not realizable in any $\mathbb{R}^{d}$. Again, the Betke-Gritzmann obstruction rules out realizability of 6-3-equivelar maps on the torus, but the obstruction was also used by McMullen, Schulz, and Wills [43] to show non-realizability for other, non-simple families of equivelar maps.

In a simplicial map (i.e., a triangulation of a surface as a simplicial complex) every triangle contains at most three odd valent vertices, from which it can be deduced that $\left|F_{W}\right| \geq|W|$ for every subset $W$ of odd valent vertices. Thus, the Betke-Gritzmann obstruction cannot be applied to simplicial maps to show non-realizability.

Grünbaum [30] proposed in 1967 that every triangulated torus should be geometrically realizable in $\mathbb{R}^{3}$. His famous conjecture was open for 40 years and was proved in 2007 by Archdeacon, Bonnington, and Ellis-Monaghan [5]. For the class of triangulations of the projective plane with one face removed geometric realizability in $\mathbb{R}^{3}$ was proved in 2008 by Bonnington and Nakamoto [16]. However, not every triangulated Möbius strip needs to be realizable in $\mathbb{R}^{3}$, a counterexample is due to by Brehm [19].

Until rather recently, no computational tools were available to actually find realizations for simplicial surfaces. In the past 30 years the most promising approach to obtain a polyhedral realization in $\mathbb{R}^{3}$ for a given triangulation was to try to build a physical model, for example, by exploiting symmetries or by employing the rubber band technique of Bokowski [11].

Very basic heuristic procedures for finding realizations in $\mathbb{R}^{3}$ for larger classes of examples were used by Fendrich [28] (to show that all triangulated tori with up to 11 vertices are realizable via embeddings in the 2-skeleta of random 4-polytopes) and Lutz [39] (to obtain realizations for triangulations of the orientable surface of genus 2 via choosing coordinates randomly). These methods, although useful in processing larger numbers of examples, are less powerful than human imagination leading to a physical model. For example, 864 of 


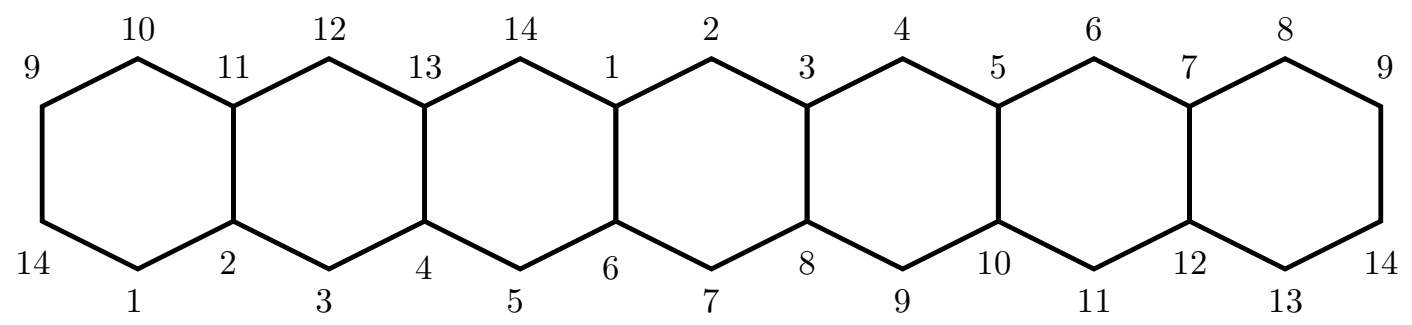

Figure 2: The non-realizable 6-3-equivelar map with 14 vertices on the torus.

the 865 vertex-minimal triangulations of the orientable surface of genus 2 were realized with the random realization method in [39], with a total computation time of 30 CPU months. However, it was not possible to randomly realize the remaining example (with the highest combinatorial symmetry of the 865 examples). The computer search was run unsuccessfully for more than a month before the example was realized within a day by Bokowski [11] with the rubber band method.

In this paper, we present a heuristic algorithm for finding polyhedral realizations for (closed, orientable) simplicial surfaces in $\mathbb{R}^{3}$, which, for the first time, surpasses the physical approach with respect to its processing time and its qualitative range of examples. In particular, we show that all vertex-minimal triangulations of the orientable surfaces of genus $g=3$ and $g=4$ are realizable. We also provide examples of vertex-minimal simplicial polyhedra of genus 5 with 12 vertices.

In the following section we give a brief survey on realizability results for surfaces, vertexminimal triangulations, and algorithmic aspects of deciding realizability. Section 3 is devoted to our realization heuristic based on the intersection segment functional. Computational results are presented in Section 4. An extension of our approach to convex realizations of triangulated spheres is discussed in Section 5.

\section{Realizability of Polyhedral Surfaces and Polyhedral Complexes}

In general, every $d$-dimensional simplicial complex (with $n$ vertices) is polyhedrally embeddable in $\mathbb{R}^{2 d+1}$, as it can be realized as a subcomplex of the boundary complex of the cyclic polytope $C(n, 2 d+2)$; cf. Grünbaum [30, Ex. 25, p. 67]. However, van Kampen [67] and Flores [29] showed that $d$-dimensional simplicial complexes cannot always be embedded topologically in $\mathbb{R}^{2 d}$, e.g., the $d$-skeleton $\operatorname{Sk}_{d}\left(\Delta_{2 d+2}\right)$ of the $(2 d+2)$-simplex $\Delta_{2 d+2}$ is not embeddable in $\mathbb{R}^{2 d}$. For further examples and references see Matoušek [42, 5.1], Novik [45], and Schild [55].

For smooth $d$-manifolds, Whitney [68] proved that they can smoothly be embedded in $\mathbb{R}^{2 d}$, and Penrose, Whitehead, and Zeeman [46] showed that for $0<2(k+1) \leq d$ every $k$ connected PL (i.e., piecewise linear) $d$-manifold has a PL embedding in $\mathbb{R}^{2 d-k}$. In particular, surfaces have PL embeddings in $\mathbb{R}^{4}$. Orientable surfaces (with or without boundary) and non-orientable surfaces with boundary are even PL embeddable in $\mathbb{R}^{3}$ (which follows from the classification of surfaces by Dehn and Heegaard [26]). Closed non-orientable $d$-manifolds cannot be embedded topologically in $\mathbb{R}^{d+1}$; cf. Bredon [17, p. 353]. 
Thus, for triangulated orientable surfaces (with or without boundary) and for triangulated non-orientable surfaces with boundary we have:

- PL embeddability in $\mathbb{R}^{3}$

- and polyhedral realizability in $\mathbb{R}^{5}$.

Triangulations of closed non-orientable surfaces are

- not (topologically) embeddable in $\mathbb{R}^{3}$,

- but are PL embeddable in $\mathbb{R}^{4}$,

- and are polyhedrally realizable in $\mathbb{R}^{5}$.

Perles showed (cf. $[30,11.1 .8]$ ) that a polyhedral map is realizable in some $\mathbb{R}^{d}$ if and only if it is realizable in $\mathbb{R}^{5}$.

A natural approach to establish geometric realizability in $\mathbb{R}^{3}$ for polyhedral maps on orientable surfaces of genus $g \geq 1$ is to identify a given polyhedral map as a subcomplex of the boundary complex of a convex 4-polytope $P$. The Schlegel diagram of $P$ then yields coordinates for the realization in $\mathbb{R}^{3}$; see, for example, McMullen, Schulz, and Wills [43] for realizations of equivelar maps obtained this way, and cf. Altshuler $[1,2]$ for combinatorial properties on maps that guarantee realizability via Schlegel diagrams.

Altshuler and Brehm [4] gave a polyhedral map $T_{8}$ on the torus with only 8 vertices which is realizable in $\mathbb{R}^{3}$ (cf. also Simutis [56]), but not via the Schlegel diagram of a convex 4-polytope. In fact, the map $T_{8}$ is not isomorphic to a subcomplex of the boundary complex of any convex polytope [4].

Realizability (via subcomplexes of convex 5-polytopes) of triangulations of the torus and the projective plane in $\mathbb{R}^{4}$ was proved by Brehm and Schild [21], herewith sharpening Barnette's result [7] on the geometric realizability of triangulations of the projective plane in $\mathbb{R}^{4}$.

Polyhedral surfaces that are obtained by projections (of 2-dimensional subcomplexes) of higherdimensional polytopes together with obstructions to projectability are discussed by Rörig, Sanyal, and Ziegler [50, 51, 52]. Knotted realizations of triangulated tori are studied by Lutz, Sullivan, and Witte in [40]. For further results and references on polyhedral maps see Brehm and Wills [23], Brehm and Schulte [22], and Ziegler [71].

\subsection{Simplicial Maps}

Let $M$ be a (closed) triangulated surface with $n=f_{0}$ vertices, $f_{1}$ edges, and $f_{2}$ triangles, i.e., $M$ has face-vector $f=\left(n, f_{1}, f_{2}\right)$. If $M$ has Euler characteristic $\chi(M)$, then by Euler's equation,

$$
n-f_{1}+f_{2}=\chi(M) .
$$

Double counting of the incidences between edges and triangles of the triangulation yields $2 f_{1}=3 f_{2}$. So together,

$$
f=(n, 3 n-3 \chi(M), 2 n-2 \chi(M)) .
$$

A triangulated surface with $n$ vertices obviously has at most $f_{1} \leq\left(\begin{array}{l}n \\ 2\end{array}\right)$ edges. By plugging in $f_{1}=3 n-3 \chi(M)$ we obtain Heawood's bound [32] from 1890 that a triangulation of a 2-manifold $M$ of Euler characteristic $\chi(M)$ has at least

$$
n \geq\left\lceil\frac{1}{2}(7+\sqrt{49-24 \chi(M)})\right\rceil
$$


vertices. Heawood's bound is sharp for all surfaces, except for the orientable surface of genus 2, the Klein bottle, and the non-orientable surface of genus 3 , where an extra vertex has to be added to the lower bound.

Corresponding vertex-minimal triangulations (i.e., triangulations with the minimal possible number of vertices) of the real projective plane $\mathbb{R} \mathbf{P}^{2}$ with 6 vertices and of the 2 -torus with 7 vertices (Möbius' torus [44]) were already known in the 19th century, but it took until 1955 to complete the construction of series of examples of vertex-minimal triangulations for all non-orientable surfaces (Ringel [49]) and until 1980 for all orientable surfaces (Jungerman and Ringel [36]).

If a given triangulation of an orientable surface is realizable in $\mathbb{R}^{3}$, then so are subdivisions of it that are obtained by successively subdividing edges and triangles. Hence, vertex-minimal triangulations apparently are good candidates for non-realizable simplicial maps. Hereby, triangulations with

$$
n=\frac{1}{2}(7+\sqrt{49-24 \chi(M)})
$$

are of particular interest (cf. [25]), as for these we have $f_{1}=\left(\begin{array}{l}n \\ 2\end{array}\right)$, that is, the respective triangulations are neighborly with complete 1-skeleton (which should make realizability difficult).

A polyhedral realization of the combinatorially unique vertex-minimal 7-vertex triangulation of the torus with $f=(7,21,14)$ was given by Császár [25], [38] (although realizability possibly was known already to Möbius; cf. [44, p. 553], [47]).

The next case of equality in (2) yields 59 examples of vertex-minimal 12-vertex triangulations of the orientable surface of genus 6 [3]; see below.

\subsection{Realizability vs. Non-Realizability of Simplicial Maps}

For every individual triangulation of an orientable surface, realizability (in $\mathbb{R}^{3}$ ) can be decided algorithmically by the following two-step procedure, cf. [10], [15, Ch. VIII]:

1. Enumerate all oriented matroids compatible with the given triangulation. If there are none, then the triangulation is not realizable, else

2. decide realizability of the oriented matroids from 1 . via solving associated polynomial inequality systems.

Theoretically, the second step can be done algorithmically (for example, with Collins' Cylindrical Algebraic Decomposition algorithm [24]). In practice, however, there are no methods known that would work sufficiently fast to yield results even for small examples. See [10] and [15, Ch. VIII] for more comments on this and on algebraic tools such as final polynomials. For general polyhedral maps on orientable surfaces Brehm proved that the realizability problem is NP-hard (as a consequence of his universality theorem for realization spaces of maps, cf. [70]). The complexity of the realization problem restricted to simplicial maps is unknown. In fact, it was open for a long time, whether there are non-realizable examples at all.

In a major breakthrough, Bokowski and Guedes de Oliveira [14] showed in 2000 (using $10 \mathrm{CPU}$ years) that one of the 59 vertex-minimal 12-vertex triangulations of the orientable surface of genus 6 has no compatible orientable matroid and therefore is not realizable.

Schewe [53, 54] substantially improved the enumeration of compatible orientable matroids and was able to verify that, in fact, all 59 vertex-minimal 12 -vertex triangulations of the orientable surface of genus 6 are non-realizable. Moreover, he found three examples of non-realizable vertex-minimal 12-vertex triangulations of the orientable surface of genus 5 . 
At least for one of these examples it is possible to remove a triangle from the triangulation while maintaining non-realizability. Connected sums with other triangulations then still are non-realizable. Hence, for every orientable surface of genus $g \geq 5$ there are triangulations that cannot be realized geometrically in $\mathbb{R}^{3}$.

Apart from the approach via oriented matroids, non-realizability results (for simplicial maps in $\mathbb{R}^{3}$ ) seem to be difficult to achieve: Novik [45] associated an integer program with a given triangulation, which, if it has no solution, yields non-realizability. Improved systems have been proposed by Timmreck [65]. So far, however, all tested systems for orientable surfaces either had solutions or turned out to be computationally intractable. In a different approach, Brehm [19] used a linking number argument to show that there is a non-realizable triangulation of the Möbius strip with 9 vertices.

\subsection{Heuristics for the Realization of Simplicial Maps}

Until recently, it was considered to be rather difficult and time-consuming to actually find realizations for given triangulations. Examples of polyhedral realizations of vertex-minimal triangulations of the orientable surfaces of genus 3 and 4 with 10 and 11 vertices, respectively, were constructed by hand by Brehm $[18,20]$ and Bokowski and Brehm $[12,13]$. Some of these examples were found by exploiting combinatorial symmetries of the triangulations, others with the rubber band technique of Bokowski [11].

A simple computer heuristic (by choosing coordinates randomly) was used in [39] to show that 864 of the 865 examples of vertex-minimal triangulations of the orientable surface of genus 2 are realizable. The remaining case then was settled by Bokowski with the rubber band method [11]. All 865 examples were later found to have realizations with small coordinates [33], i.e., all these examples are realizable with integer coordinates in general position in the $(4 \times 4 \times 4)$-cube. Moreover, realizations in the $(5 \times 5 \times 5)$-cube were obtained for 17 of the 20 vertex-minimal triangulations with 10 vertices of the orientable surface of genus 3 by isomorphism-free enumeration of possible coordinate configurations in general position [34].

In the following, we will discuss an improved heuristic to obtain polyhedral realizations in $\mathbb{R}^{3}$ for triangulations of orientable surfaces. In particular, we will show that all vertexminimal triangulations of the orientable surfaces of genus $g=3$ and $g=4$ are realizable and that there are examples of simplicial polyhedra of genus 5 with 12 vertices.

\section{Realization with the Intersection Segment Functional}

As mentioned in the previous section there have been so far three major heuristics for the realization of simplicial surfaces (of genus $g \geq 1$ ) in $\mathbb{R}^{3}$ :

- by explicit geometric construction $[12,13,18,20]$ (e.g., via the rubber band technique of Bokowski [11]);

- by choosing coordinates randomly [39];

- by enumeration of realizations with small coordinates [33, 34, 35].

As a more sophisticated approach we suggest to proceed as follows. For a given triangulation (of an orientable surface of small genus with few vertices)

1. start with random coordinates for the vertices of the triangulation

2. and then "move vertices around" to eventually obtain a realization. 


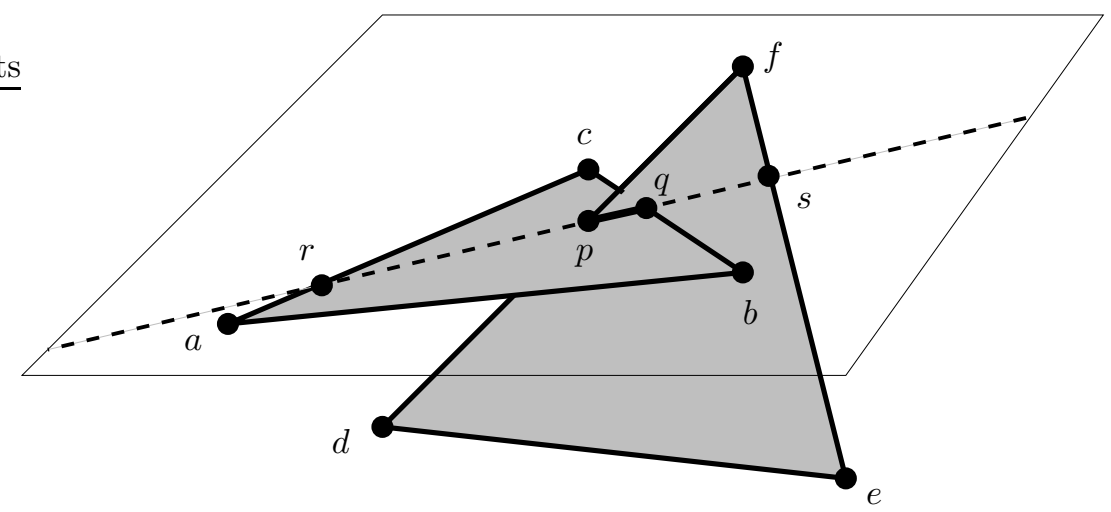

Figure 3: Two intersecting triangles.

For the second step we take as an objective to minimize the intersection segment functional:

Let $M^{2}$ be a triangulated orientable surface with vertex-set $V$ and let $V_{\mathbb{Z}^{3}}$ be a set of $|V|$ integer vertices in general position in $\mathbb{R}^{3}$. Then every pair of triangles of $M^{2}$ coordinatized with the coordinates of $V_{\mathbb{Z}^{3}}$ either has empty intersection in $\mathbb{R}^{3}$ or intersects in a segment; see Figure 3 for the intersection segment $p-q$ of two triangles. The sum of the lengths of the intersection segments over all pairs of (non-neighboring) triangles is the intersection segment functional.

We require that the points are in general position, i.e., no three points are on a line and no four points are on a plane, in order to avoid degenerate intersections of triangles. Further, we use integer coordinates and therefore move the points in the second step above on the integer grid $\mathbb{Z}^{3}$ only. Our aim then will be to find integer coordinates in general position for which the intersection segment functional vanishes for the given triangulation.

From an initial set of random coordinates we proceed to minimize the intersection segment functional by a local search of hill-climbing type:

In every step, we randomly pick a vertex $v \in V_{\mathbb{Z}^{3}}$ and a coordinate direction, $\pm x, \pm y$, or $\pm z$, and then move the vertex $v$ one integer step into the respective direction. If the resulting set of coordinates is in general position and the new value of the intersection segment functional is strictly smaller than before, the move is accepted and the next step is executed. Otherwise the move is discarded and we start anew from the previous set of coordinates.

If all possible choices of moves have been tested for some set of coordinates without improvement, then we are stuck in a local minimum. In this case, for one step only, we choose one of the admissible moves, i.e., a move that yields a set of coordinates in general position, but which not necessarily decreases the intersection segment functional. From there, we then try to continue to decrease the intersection segment functional in a new direction. 


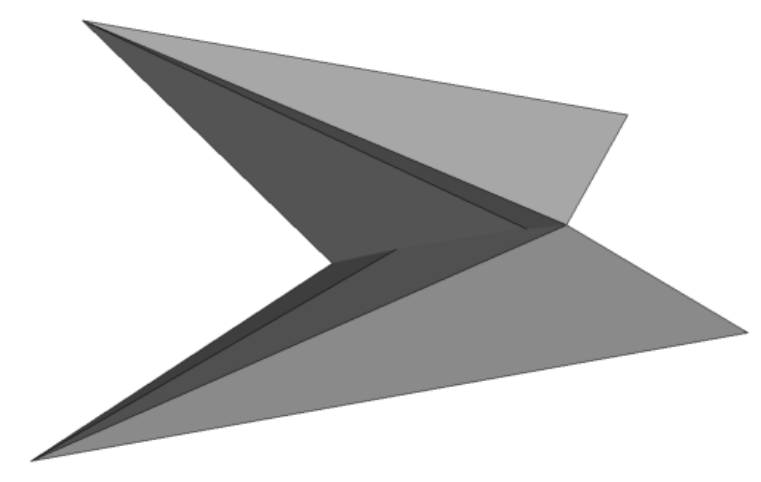

Figure 4: A "non-realization" of the octahedron with locally minimal functional.

Example 3: Local minima with positive value of the intersection segment functional can occur even for small triangulations. For example, the boundary of the octahedron with triangles

$$
\begin{array}{llllllll}
123 & 124 & 135 & 145 & 236 & 246 & 356 & 456
\end{array}
$$

and furnished with coordinates (in general position)

$$
\text { 1: }(4,4,6) \quad 2:(5,6,6) \quad 3:(9,7,4) \quad 4:(5,9,1) \quad 5:(4,6,3) \quad 6:(1,5,7)
$$

attains a local minimum for the intersection segment functional with value 3.17; see Figure 4 for a visualization.

\subsection{Details of the Algorithm}

Initially the vertices of the triangulation are placed randomly at general positions in a cube of size $50 \times 50 \times 50$. This cube is chosen at the center of a larger $(250 \times 250 \times 250)$-cube that we take as bounding box for all possible positions of the vertices during the local search. After the choice of the starting positions the smaller cube is not used anymore.

- Thus, we allow the diameter of the vertex-set to increase moderately (which possibly helps to decrease the intersection segment functional by unfolding the initial shape).

- At the same time there is a fixed lower bound for the change, at every step, of the intersection segment functional (determined by the size of the bounding box and the fact that we admit integer coordinates only). This way we avoid that the sequence of improvements for the functional converges to zero.

An admissible step then is a movement of one vertex by one integer in one of the coordinate directions such that the resulting set of coordinates is in general position and is within the bounding box.

- If the intersection segment functional becomes zero, a realization for the given triangulation is found.

- If a realization is not found within a fixed period of time $T$, the whole process is restarted for the triangulation, beginning with the random selection of the starting coordinates (in the smaller cube). In doing so we try to overcome situations in which the process cycles between different local minima. 

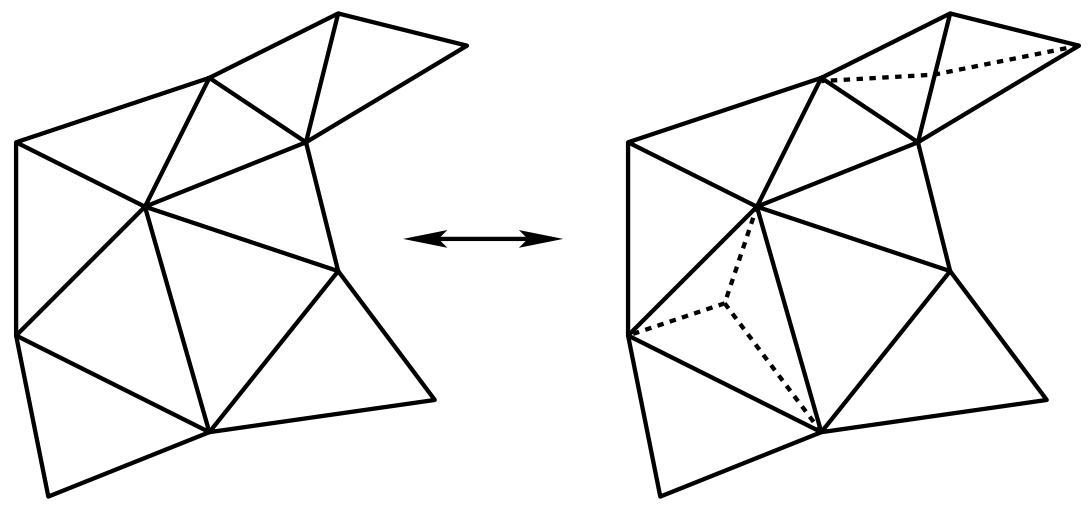

Figure 5: Subdivisions of a triangle and of an edge.

A standard problem with local search algorithms is to appropriately choose the parameters that govern the procedure. For some of the 20 examples of vertex-minimal 10-vertex triangulations of the orientable surface of genus 3 we tried the following variants:

- We chose different sizes for the initial cube, ranging from $5 \times 5 \times 5$ to $500 \times 500 \times 500$.

- We allowed the bounding box to be between 1 up to 8 times the size of the initial cube.

- If the segment functional decreases by moving one vertex in one direction, we moved the vertex as far as possible in this direction (until the intersection segment functional starts to increase again).

- In case of a local minimum we determined all pairs of vertices for which the exchange of their positions decreases the intersection segment functional. We then executed one such exchange at random. If there is no such pair, we randomly exchanged two arbitrary vertices.

- Instead of minimizing the intersection segment functional we tried to minimize the normalized intersection segment functional, which is obtained from the intersection segment functional by dividing by the total length of the edges of the coordinatized triangulation.

- We first generated 10000 sets of initial coordinates of which we selected the set with the smallest functional before starting the local search.

From all these variants the previously described one turned out to have the best performance. This variant then was used to find realizations for other triangulations.

\subsection{Test Sets of Minimal Triangulations}

If some triangulation of an orientable surface is realizable, then so are all subdivisions of it that result from the starting triangulation by an iterative sequence of elementary subdivisions of triangles and edges (see Figure 5). For every geometrically realized triangulation in such a sequence, we can always place the new vertex slightly "above" or "below" the respective triangle or the respective edge of the previous realization. Alternatively, we could choose all new vertices on the original surface and then slightly perturb the coordinates of the new vertices into general position. 


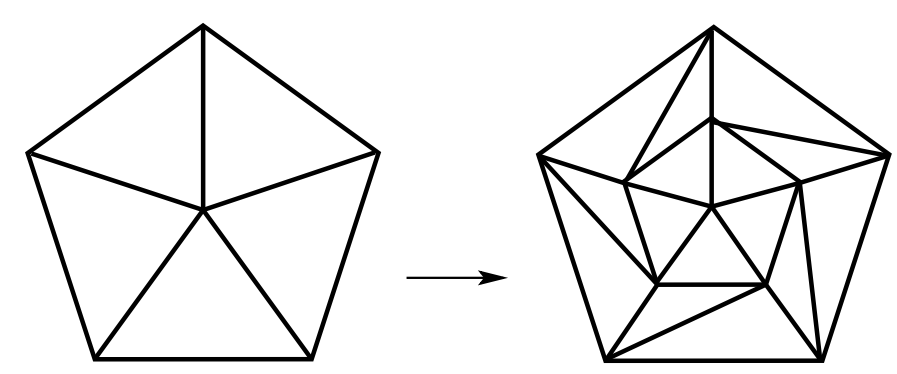

Figure 6: Replacement of the star of a vertex of degree 5.

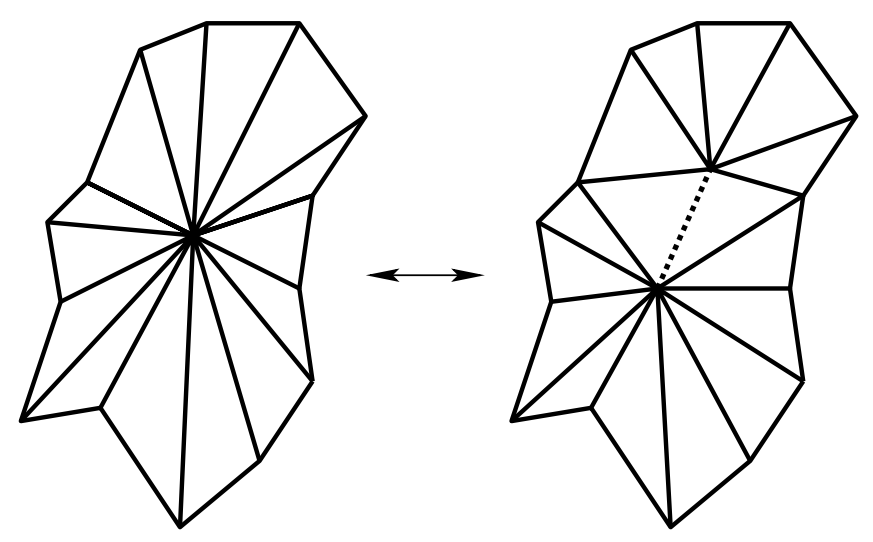

Figure 7: Expansion (respectively contraction) of an edge.

A triangulation of a surface is minimal if it does not result from a triangulation with fewer vertices by a sequence of elementary subdivisions. If all minimal triangulations of a surface are realizable, then all triangulations of the surface are realizable. Unfortunately, for surfaces of genus $g \geq 1$ the set of minimal triangulations is infinite: it comprises the infinite set of triangulations with all vertices of degree at least 5 , since for any such triangulation we can replace the star of a vertex (i.e., all triangles that contain the vertex) with a patch that has more vertices, but all of degree at least 5; see Figure 6. Equivelar triangulations of surfaces of genus $g \geq 1$ are minimal. For $g=1$ there are infinitely many equivelar triangulations, whereas for $g>1$ there are only finitely many examples; cf. [63].

A finite subset (of the set of minimal triangulations) of particular interest to test realizability is the set of vertex-minimal triangulations. If these are realizable, then this should give a strong indication that, in fact, all triangulations of the surface are realizable.

A larger, but still finite set of minimal triangulations that contains all vertex-minimal triangulations is defined as follows: If we allow edge expansions (with edge contractions as inverses; see Figure 7) instead of elementary subdivisions, then for every surface there is only a finite set (see Barnette and Edelson [8]) of irreducible triangulations for which no edge can be contracted without changing the topological type of the triangulation. Unfortunately, it is, a priori, not clear whether a realizable triangulation of an orientable surface of genus $g \geq 1$ remains realizable after the expansion of an edge. At least, for every explicit polyhedral 
Table 1: Numbers of vertex-minimal triangulations of the orientable surfaces of genus $g \leq 6$.

\begin{tabular}{rrr}
\hline$g$ & $n_{\text {min }}$ & Types \\
\hline 0 & 4 & 1 \\
1 & 7 & 1 \\
2 & 10 & 865 \\
3 & 10 & 20 \\
4 & 11 & 821 \\
5 & 12 & 751.593 \\
6 & 12 & 59 \\
\hline
\end{tabular}

realization it can easily be tested whether a particular edge expansion can be carried out (via a system of linear constraints on the link of the respective vertex; we thank the anonymous referee for pointing this out to us).

It follows from the work of Steinitz $[58, \S 46]$ that every triangulated 2-sphere can be reduced to the boundary of the tetrahedron by a sequence of edge contractions, that is, the boundary of the tetrahedron is the only irreducible triangulation of the 2-sphere. Grünbaum and Lavrenchenko [37] determined the number of irreducible triangulations of the torus: there are 21 such examples with up to 10 vertices and they are all realizable. Sulanke [60, 61, 62] showed by enumeration that there are exactly 396.784 examples of irreducible triangulations (with up to 17 vertices) of the orientable surface of genus 2 .

Although it might be desirable to test realizability for a larger set of irreducible triangulations, we restricted ourselves to vertex-minimal ones. There is only one unique vertex-minimal triangulation of the torus, i.e., Möbius 7-vertex torus [44] for which Császár [25] gave an explicit polyhedral model. Vertex-minimal triangulations of the orientable surfaces of genus 2 and 3 were enumerated in [39], those of genus 4 and 5 in [63], and the vertex-minimal examples of genus 6 in [3]; see Table 1 for the corresponding minimal numbers of vertices $n_{\min }$ and the respective numbers of combinatorial types of triangulations.

\section{Computational Results}

\section{Genus 2}

In [39], geometric realizations for 864 of the 865 vertex-minimal 10-vertex triangulations of the orientable surface of genus 2 were found with the random realization approach in a total computation time of $30 \mathrm{CPU}$ months on a $2.8 \mathrm{GHz}$ processor; the remaining example then was realized with the rubber band method [11]. For realizations of the 865 examples with small coordinates see [33] and the comments above. With our new heuristic algorithm, based on the intersection segment functional, realizations for the 865 triangulations were obtained in a total time of $218 \mathrm{CPU}$ minutes on a $3.5 \mathrm{GHz}$ processor. 


\section{Genus 3}

Realizations for 5 of the 20 vertex-minimal 10-vertex triangulations of the orientable surface of genus 3 were constructed by hand by Brehm and Bokowski $[12,18,20]$. The random realization approach of [39], however, produced no results for these 5 (and for the other 15) examples, where we stopped the search after one CPU week each. Therefore, the basic random realization approach is not suitable for triangulations of surfaces of higher genus (or with more vertices). For 17 of the 20 triangulations realizations with small coordinates in the $(5 \times 5 \times 5)$-cube were obtained in [34]; this search was run (in total) for 2 CPU years on a $3.5 \mathrm{GHz}$ processor. Thus, the first task for our new program was to realize the remaining three examples.

Theorem 1 All 20 vertex-minimal 10-vertex triangulations of the orientable surface of genus 3 are geometrically realizable in $\mathbb{R}^{3}$.

Sets of coordinates for the realizations are available online at $[34,41]$. In total, it took $28 \mathrm{CPU}$ hours on a $3.5 \mathrm{GHz}$ processor to realize the 20 examples with the help of the intersection segment functional. For two of the last three of the 20 examples, we later found realizations in the $(6 \times 6 \times 6)$-cube; cf. [41].

\section{Genus 4}

A first example of a polyhedron of genus 4 with 11 vertices was described by Bokowski and Brehm [13]. With our intersection segment functional algorithm we found realizations for 626 of the 821 vertex-minimal 11-vertex triangulations of the orientable surface of genus 4 . In an effort to speed up the search, realizations for the remaining 195 triangulations were obtained by recycling of coordinates, that is, whenever a new realizations was found we tried to reuse the respective set of coordinates for other triangulations. We also slightly distorted the coordinates and then tried to use these coordinates for other triangulations; see [39] for additional comments.

Theorem 2 All 821 vertex-minimal 11-vertex triangulations of the orientable surface of genus 4 are geometrically realizable in $\mathbb{R}^{3}$.

We needed a total of $9.51 \cdot 10^{11}$ steps of the local search process to realize the 626 triangulations. As time interval $T$ we chose 15 minutes, so if after 15 minutes (about $5.4 \cdot 10^{6}$ steps) a realization was not reached, the search was restarted with new initial coordinates.

Figure 8 displays a histogram of the natural logarithms of the number of used steps. The picture indicates that the logarithms of the used steps are normally distributed, i.e., the used steps underlie a log-normal distribution. To confirm this, we ran as a goodness-of-fit-test [59, Ch. 30] the Anderson-Darling test (cf. [27, p. 10]). The test estimates the mean to be 19.3 and the standard deviation to be 2 . It yields a $p$-value of 0.5 , which is far above the rejection value of 0.05 . Therefore we can view the logarithms of the used steps to be normally distributed with the estimated parameters.

Our implementation of the local search process is performing about $3.6 \cdot 10^{5}$ steps per minute on a $3.5 \mathrm{GHz}$ processor. Therefore, we needed a total of $5 \mathrm{CPU}$ years to realize all triangulations. On average, it took 2.9 CPU days for finding a realization for a single triangulation. 


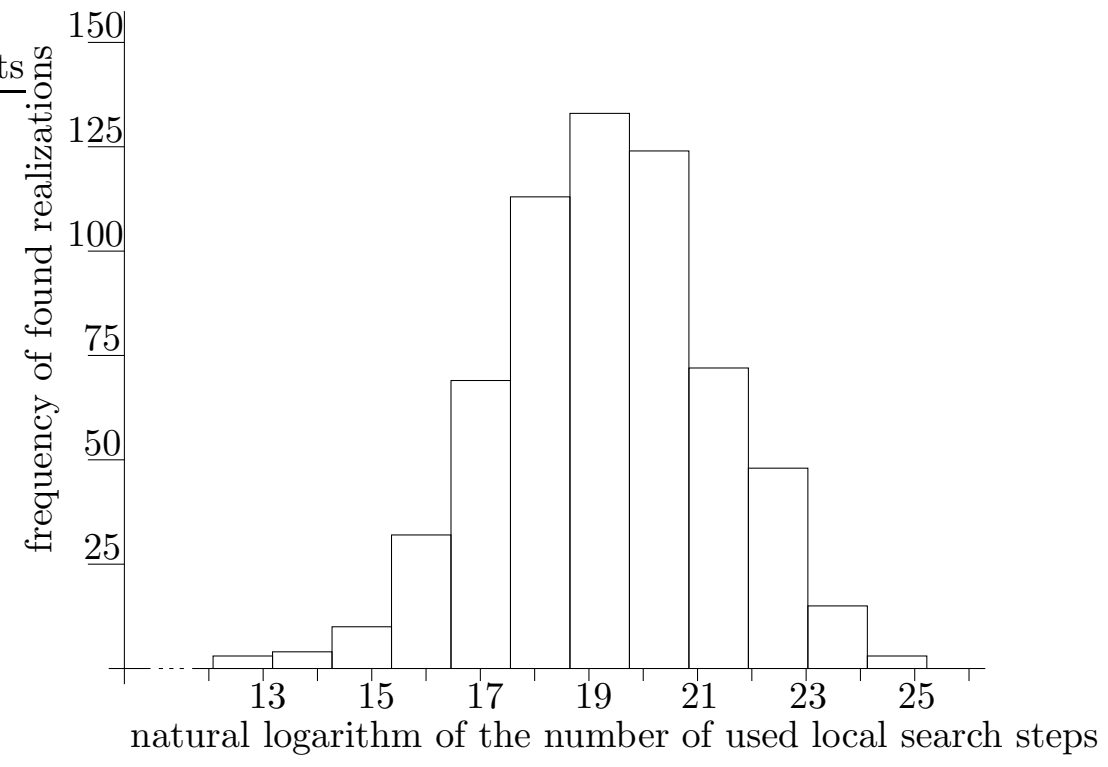

Figure 8: Histogram of the natural logarithms of the used local search steps for 626 realizations of genus 4 .

\section{Genus 5}

As mentioned in Section 2, Schewe $[53,54]$ showed that there are at least three examples of vertex-minimal 12-vertex triangulations of the orientable surface of genus 5 that cannot be realized geometrically in $\mathbb{R}^{3}$.

In order to complement Schewe's result, we tried to find realizations for at least some of the 751.593 triangulations. To this aim we started our process on randomly selected triangulations out of all the 751.593 vertex-minimal triangulations. If after 15 minutes a realization was not found, a new triangulation was selected at random. This way, we tried about 94.000 triangulations, using a total of $7.52 \cdot 10^{11}$ local search steps - a CPU time of approximately 4 years - and succeeded in realizing 15 triangulations.

Theorem 3 At least 15 of the 751.593 vertex-minimal 12-vertex triangulations of the orientable surface of genus 5 are geometrically realizable in $\mathbb{R}^{3}$.

Since the 94.000 triangulations we tested were chosen randomly from the list of the 751.593 genus 5 triangulations, probably at least 120 (and perhaps many more) of the examples are realizable.

Example 4: Figure 9 displays one of the polyhedra of genus 5 with 12 vertices, which has triangles

$\begin{array}{llllllllll}123 & 124 & 135 & 146 & 157 & 168 & 179 & 1810 & 1910 & 236 \\ 245 & 258 & 2610 & 2811 & 2911 & 2912 & 21012 & 3511 & 368 & 378 \\ 3710 & 3910 & 3911 & 459 & 4611 & 478 & 4712 & 489 & 41011 & 41012 \\ 569 & 5610 & 5710 & 5812 & 51112 & 679 & 6712 & 61112 & 8912 & 81011\end{array}$




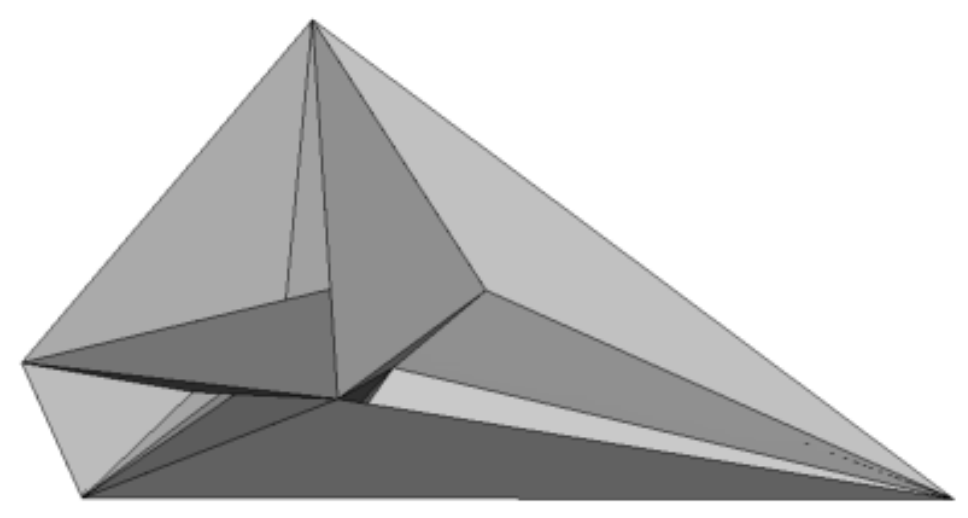

Figure 9: A polyhedron of genus 5.

and coordinates

\begin{tabular}{|c|c|c|c|}
\hline$(137,124,141)$ & $(107,118,143)$ & $(132,130,125)$ & $(122,127,129)$ \\
\hline & $(126,130,124)$ & $(126,129,129)$ & $(122,125,13$ \\
\hline$(124,128,136)$ & 10: $(119,133,134)$ & 11: $(120,130,135)$ & 12: $\quad(121,128,13$ \\
\hline
\end{tabular}

The coordinates for the other 14 examples can be found online at [41].

Combining the result of Schewe $[53,54]$ (that there are non-realizable triangulations of the orientable surface of genus 5 ) with our finding (that all vertex-minimal triangulations of surfaces of genus $g \leq 4$ are realizable) gives rise to:

Conjecture 4 Every triangulation of an orientable surface of genus $g \leq 4$ is geometrically realizable.

The conjecture holds for genus 0 (Steinitz $[57,58]$ ) and for genus 1 (Archdeacon, Bonnington, and Ellis-Monaghan [5]).

\subsection{Examples with More Vertices}

We also tried our program on some triangulations of tori with more vertices. It turned out that it still is possible to find realizations, although it takes much longer for every step of the local search process: There are $O\left(|V|^{2}\right)$ pairs of triangles that have to be considered for the computation, respectively for the update, of the intersection segment functional. Moreover, there are $6|V|$ possible moves from a current set of coordinates that lead to a new set of coordinates. In the worst case, we are forced to test almost all these moves just to carry out a single improvement step. Finally, the initial value of the intersection segment functional will be larger for triangulations with more vertices, thus, forcing us to perform more steps. 


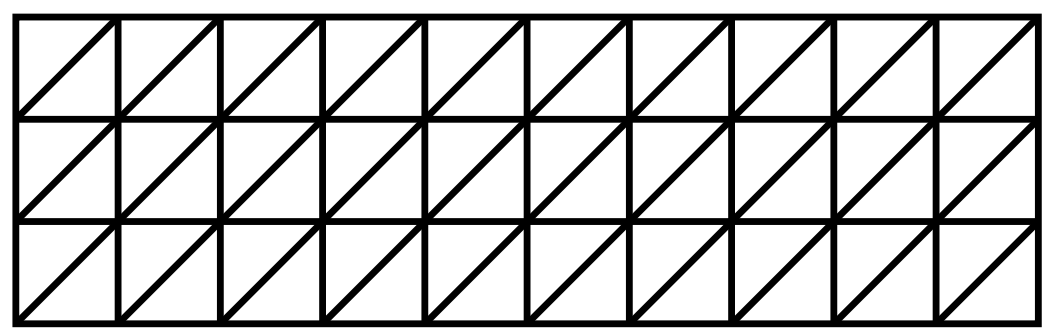

Figure 10: The standard $(3 \times 10)$-torus.
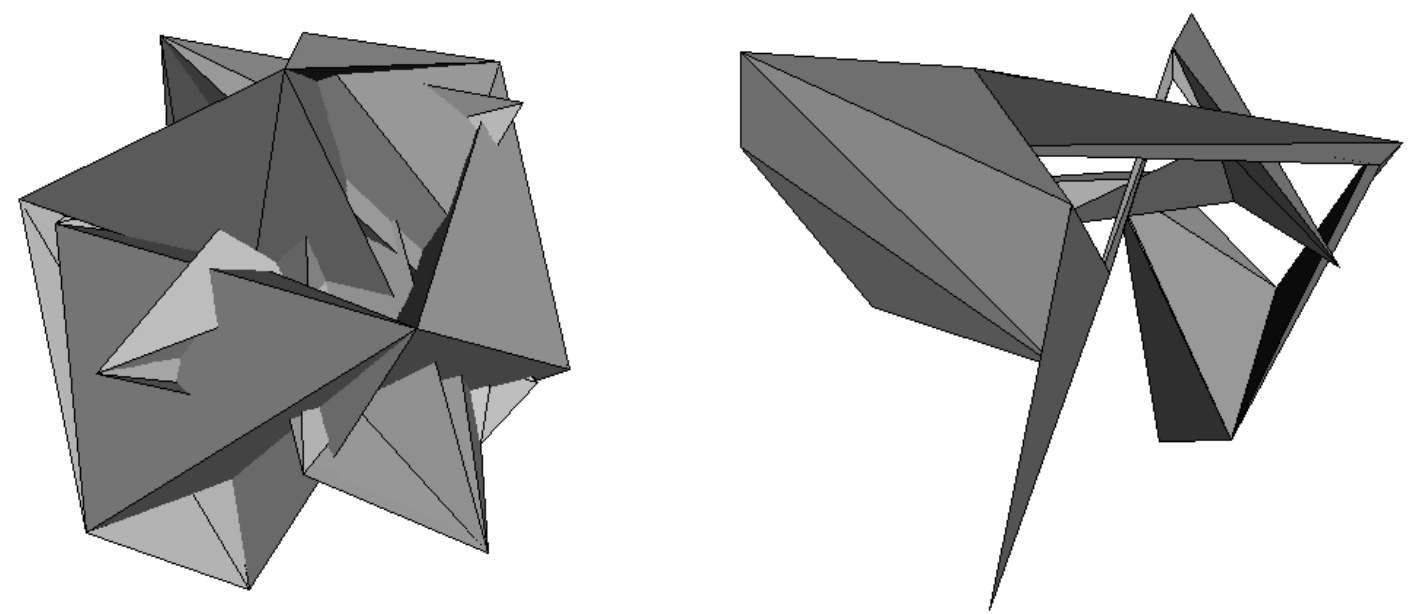

Figure 11: The standard $(3 \times 10)$-torus with random coordinates and a proper realization.

Example 5: For the standard $(3 \times 10)$-torus (Figure 10) we started with random coordinates (Figure 11, left) and an initial value 7924.26 of the intersection segment functional. It then took 3042 local search steps to obtain a proper realization (Figure 11, right).

\section{Convex Realizations of Triangulated 2-Spheres}

According to Steinitz [57, 58], every polyhedral map on the 2-sphere $S^{2}$ is geometrically realizable in $\mathbb{R}^{3}$ as the boundary complex of a convex 3-polytope. Tutte's equilibrium method [66] (see also [48, Section 12.2]) allows to obtain corresponding realizations algorithmically via first constructing a planar equilibrium representation of the edge graph of a given map. The resulting planar graph can then be interpreted as the Schlegel diagram of a 3-polytope.

Triangulated 2-spheres are realizable as boundary complexes of simplicial 3-polytopes. However, simplicial 3- and higher-dimensional spheres need not be polytopal. The BrücknerGrünbaum 3-sphere [31] and the Barnette 3-sphere [6], both with 8-vertices, are the smallest examples of non-polytopal simplicial spheres. 
In the following, we give a simple modification of our realization heuristic in order to obtain convex realizations of triangulated 2-spheres. (By using an intersection area functional one might generalize this approach to search for convex realizations of simplicial 3-spheres in $\mathbb{R}^{4}$.)

A non-face of a triangulated 2-sphere with $n$ vertices is a two-element subset (an edge) or a three-element subset (a triangle) of the ground set of $n$ vertices that does not constitute a face of the triangulation. In any convex realization (in general position) of the triangulated 2 -sphere as the boundary complex of a simplicial 3-polytope, every non-face intersects with the interior of the respective polytope. In particular, every face of the boundary 2-sphere either has no intersection with a given non-face or intersects the non-face in an edge or a vertex of the boundary sphere.

Hence, by adding to the intersection segment functional the lengths of intersection segments for all pairs of triangles consisting of a triangle of the triangulation and a triangle that does not belong to the triangulation the resulting extended intersection segment functional can be used to obtain convex realizations for triangulated 2-spheres. To be more precise:

Proposition 5 If a triangulated 2-sphere has no vertex of degree 3 , then the extended intersection segment functional is zero if and only if a convex realization (with vertices in general positions) has been reached.

Proof. If a convex realization has been reached, then obviously the extended intersection segment functional is zero.

For the other direction, assume that the functional is zero and that the vertices are in general position. In case some vertex $v$ is contained in the convex hull of the other $n-1$ vertices, then $v$ is contained in the convex hull of some subset $\left\{v_{1}, v_{2}, v_{3}, v_{4}\right\}$ of four of the $n-1$ vertices: Pick any vertex $v_{1}$ on the boundary of the convex hull of the $n$ vertices, then by the general position assumption, there is a unique triangle $\left\{v_{2}, v_{3}, v_{4}\right\}$ (opposite to $v_{1}$ with respect to $v$ ) on the boundary of the convex hull such that the tetrahedron $\left\{v_{1}, v_{2}, v_{3}, v_{4}\right\}$ contains $v$. Without loss of generality, we may assume that no other of the $n-1$ vertices is contained in the tetrahedron $\left\{v_{1}, v_{2}, v_{3}, v_{4}\right\}$ : If there is an additional such vertex, say, $v^{\prime}$, then the convex hull of $v^{\prime}$ with the triangle on the boundary of the tetrahedron $\left\{v_{1}, v_{2}, v_{3}, v_{4}\right\}$ opposite to $v^{\prime}$ with respect to $v$ is a tetrahedron (of smaller volume) that contains $v$. Moreover, since the smallest example of a triangulated 2-sphere without a vertex of degree 3 is the boundary complex of the octahedron with 6 vertices, there is at least one vertex of the triangulation that lies outside the tetrahedron $\left\{v_{1}, v_{2}, v_{3}, v_{4}\right\}$. If the vertex $v$ has degree larger than 4 , then at least one of the triangles of the star of $v$ intersects non-trivially some boundary triangle (a face or a non-face of the triangulation) of the tetrahedron $\left\{v_{1}, v_{2}, v_{3}, v_{4}\right\}$. The line segment the two triangles intersect in contributes a positive value to the extended intersection segment functional, contradiction. If $v$ has degree 4 , then there are two cases. If the star of the vertex $v$ contains a vertex different from $v_{1}, v_{2}, v_{3}, v_{4}$, then again at least one of the triangles in the star of $v$ intersects some triangle of the boundary of the tetrahedron $\left\{v_{1}, v_{2}, v_{3}, v_{4}\right\}$, contradiction. Else, let $v_{5}$ be a vertex outside the convex hull of the vertices $v_{1}, v_{2}, v_{3}, v_{4}$. Then $v$ lies in the convex hull of $v_{5}$ and some triangle $\left\{v_{i_{1}}, v_{i_{2}}, v_{i_{3}}\right\}$ of the tetrahedron $\left\{v_{1}, v_{2}, v_{3}, v_{4}\right\}$. But then the vertex star of $v$ contains the vertex $v_{4}$, which lies outside the tetrahedron spanned by the vertices $v_{i_{1}}, v_{i_{2}}, v_{i_{3}}, v_{5}$. This again leads to a contradiction. 
In case a triangulation has vertices of degree 3 , non-convex realizations of the triangulation can have vanishing extended intersection segment functional. The smallest such example is the bipyramid over a triangle with one apex pushed inside the convex hull of the other four vertices.

Nevertheless, we can recursively remove vertices of degree 3 from a given triangulation. The resulting triangulation then either is the boundary of a tetrahedron or a triangulation with vertices all of degree at least four. After obtaining a realization for the simplified triangulation, the removed vertices can be added back by placing them suitably "above" the triangles which they subdivide.

We successfully tested our approach for some small triangulations of $S^{2}$ : there are 233 triangulations of $S^{2}$ with 10 vertices of which 12 examples have no vertices of degree 3 . It took, on average, about 5 minutes to obtain convex realizations for these 12 triangulations.

Remark: Although our main focus in this paper was on the realization of closed, orientable triangulated surfaces, the intersection segment functional can, of course, also be used to search for realizations in three-space for other 2-dimensional simplicial complexes. Moreover, the functional can easily be modified for a search for immersions of (orientable or non-orientable) triangulated surfaces.

\section{Acknowledgment}

We are grateful to the anonymous referee for many helpful comments.

\section{References}

[1] A. Altshuler. Manifolds in stacked 4-polytopes. J. Comb. Theory, Ser. A 10, 198-239 (1971).

[2] A. Altshuler. Polyhedral realization in $\mathbb{R}^{3}$ of triangulations of the torus and 2-manifolds in cyclic 4-polytopes. Discrete Math. 1, 211-238 (1971).

[3] A. Altshuler, J. Bokowski, and P. Schuchert. Neighborly 2-manifolds with 12 vertices. J. Comb. Theory, Ser. A 75, 148-162 (1996).

[4] A. Altshuler and U. Brehm. A non-Schlegelian polyhedral map on the torus. Mathematika 31, 83-88 (1984).

[5] D. Archdeacon, C. P. Bonnington, and J. A. Ellis-Monaghan. How to exhibit toroidal maps in space. Discrete Comput. Geom. 38, 573-594 (2007).

[6] D. Barnette. The triangulations of the 3 -sphere with up to 8 vertices. J. Comb. Theory, Ser. A 14, 37-52 (1973).

[7] D. Barnette. All triangulations of the projective plane are geometrically realizable in $E^{4}$. Isr. J. Math. 44, 75-87 (1983).

[8] D. W. Barnette and A. L. Edelson. All 2-manifolds have finitely many minimal triangulations. Isr. J. Math. 67, 123-128 (1988). 
[9] U. Betke and P. Gritzmann. A combinatorial condition for the existence of polyhedral 2-manifolds. Isr. J. Math. 42, 297-299 (1982).

[10] J. Bokowski. Effective methods in computational synthetic geometry. Automated Deduction in Geometry, Proc. 3rd Internat. Workshop (ADG 2000), Zürich, 2000 (J. RichterGebert and D. Wang, eds.). Lecture Notes in Computer Science 2061, 175-192. SpringerVerlag, Berlin, 2001.

[11] J. Bokowski. On heuristic methods for finding realizations of surfaces. Discrete Differential Geometry (A. I. Bobenko, P. Schröder, J. M. Sullivan, and G. M. Ziegler, eds.). Oberwolfach Seminars 38, 255-260. Birkhäuser, Basel, 2008.

[12] J. Bokowski and U. Brehm. A new polyhedron of genus 3 with 10 vertices. Intuitive Geometry, Internat. Conf. on Intuitive Geometry, Siófok, Hungary, 1985 (K. Böröczky and G. Fejes Tóth, eds.). Colloquia Mathematica Societatis János Bolyai 48, 105-116. North-Holland, Amsterdam, 1987.

[13] J. Bokowski and U. Brehm. A polyhedron of genus 4 with minimal number of vertices and maximal symmetry. Geom. Dedicata 29, 53-64 (1989).

[14] J. Bokowski and A. Guedes de Oliveira. On the generation of oriented matroids. Discrete Comput. Geom. 24, 197-208 (2000).

[15] J. Bokowski and B. Sturmfels. Computational Synthetic Geometry. Lecture Notes in Mathematics 1355. Springer-Verlag, Berlin, 1989.

[16] C. P. Bonnington and A. Nakamoto. Geometric realization of a triangulation on the projective plane with one face removed. Discrete Comput. Geom. 40, 141-157 (2008).

[17] G. E. Bredon. Topology and Geometry. Graduate Texts in Mathematics 139. SpringerVerlag, New York, NY, corrected third printing, 1997 edition, 1993.

[18] U. Brehm. Polyeder mit zehn Ecken vom Geschlecht drei. Geom. Dedicata 11, 119-124 (1981).

[19] U. Brehm. A nonpolyhedral triangulated Möbius strip. Proc. Am. Math. Soc. 89, 519$522(1983)$.

[20] U. Brehm. A maximally symmetric polyhedron of genus 3 with 10 vertices. Mathematika 34, 237-242 (1987).

[21] U. Brehm and G. Schild. Realizability of the torus and the projective plane in $\mathbb{R}^{4}$. Isr. J. Math. 91, 249-251 (1995).

[22] U. Brehm and E. Schulte. Polyhedral maps. Handbook of Discrete and Computational Geometry (J. E. Goodman and J. O'Rourke, eds.), Chapter 18, 345-358. CRC Press, Boca Raton, FL, 1997.

[23] U. Brehm and J. M. Wills. Polyhedral manifolds. Handbook of Convex Geometry, Volume A (P. M. Gruber and J. M. Wills, eds.), Chapter 2.4, 535-554. North-Holland, Amsterdam, 1993. 
[24] G. E. Collins. Quantifier elimination for real closed fields by cylindrical algebraic decompositon. Automata Theory and Formal Languages, 2nd GI conference, Kaiserslautern, 1975 (H. Brakhage, ed.). Lecture Notes in Computer Science 33, 134-183. SpringerVerlag, Berlin, 1975.

[25] A. Császár. A polyhedron without diagonals. Acta Sci. Math., Szeged 13, 140-142 (1949-1950).

[26] M. Dehn and P. Heegaard. Analysis situs. Encyklopädie der mathematischen Wissenschaften mit Einschluss ihrer Anwendungen, Dritter Band: Geometrie, III.1.1., Heft 1 (W. Fr. Meyer and H. Mohrmann, eds.), Chapter III A B 3, 153-220. B. G. Teubner, Leipzig, 1907.

[27] B. S. Everitt. The Cambridge Dictionary of Statistics. Cambridge University Press, Cambridge, 1998.

[28] S. Fendrich. Methoden zur Erzeugung und Realisierung von triangulierten kombinatorischen 2-Mannigfaltigkeiten. Diplomarbeit, Technische Universität Darmstadt, 2003, 56 pages.

[29] A. I. Flores. Über die Existenz $n$-dimensionaler Komplexe, die nicht in den $\mathbb{R}^{2 n}$ topologisch einbettbar sind. Erg. Math. Kolloqu. 5, 17-24 (1933).

[30] B. Grünbaum. Convex Polytopes. Pure and Applied Mathematics 16. Interscience Publishers, London, 1967. Second edition (V. Kaibel, V. Klee, and G. M. Ziegler, eds.), Graduate Texts in Mathematics 221. Springer-Verlag, New York, NY, 2003.

[31] B. Grünbaum and V. P. Sreedharan. An enumeration of simplicial 4-polytopes with 8 vertices. J. Comb. Theory 2, 437-465 (1967).

[32] P. J. Heawood. Map-colour theorem. Quart. J. Pure Appl. Math. 24, 332-338 (1890).

[33] S. Hougardy, F. H. Lutz, and M. Zelke. Polyhedra of genus 2 with 10 vertices and minimal coordinates. Electronic Geometry Models No. 2005.08.001 (2007). http://www . eg-models.de/2005.08.001.

[34] S. Hougardy, F. H. Lutz, and M. Zelke. Polyhedra of genus 3 with 10 vertices and minimal coordinates. Electronic Geometry Models No. 2006.02 .001 (2007). http://www . eg-models.de/2006.02.001.

[35] S. Hougardy, F. H. Lutz, and M. Zelke. Polyhedral tori with minimal integer coordinates. Electronic Geometry Models No. 2008.10.001 (2008). http://www.eg-models.de/2008. 10.001.

[36] M. Jungerman and G. Ringel. Minimal triangulations on orientable surfaces. Acta Math. 145, 121-154 (1980).

[37] S. A. Lavrenchenko. Irreducible triangulations of the torus. J. Sov. Math. 51, 2537-2543 (1990). Translation from Ukr. Geom. Sb. 30, 52-62 (1987). 
[38] F. H. Lutz. Császár's torus. Electronic Geometry Models No. 2001.02 .069 (2002). http: //www.eg-models.de/2001.02.069.

[39] F. H. Lutz. Enumeration and random realization of triangulated surfaces. Discrete Differential Geometry (A. I. Bobenko, P. Schröder, J. M. Sullivan, and G. M. Ziegler, eds.). Oberwolfach Seminars 38, 235-253. Birkhäuser, Basel, 2008.

[40] F. H. Lutz and N. Witte. Knotted polyhedral tori. arXiv:0707.1281, 2007, 10 pages.

[41] F. H. Lutz. The Manifold Page, 1999-2009. http://www.math.tu-berlin.de/ diskregeom/stellar/.

[42] J. Matoušek. Using the Borsuk-Ulam Theorem. Lectures on Topological Methods in Combinatorics and Geometry. Universitext. Springer-Verlag, Berlin, 2003.

[43] P. McMullen, Ch. Schulz, and J. M. Wills. Equivelar polyhedral manifolds in $E^{3}$. Isr. J. Math. 41, 331-346 (1982).

[44] A. F. Möbius. Mittheilungen aus Möbius' Nachlass: I. Zur Theorie der Polyëder und der Elementarverwandtschaft. Gesammelte Werke II (F. Klein, ed.), 515-559. Verlag von S. Hirzel, Leipzig, 1886.

[45] I. Novik. A note on geometric embeddings of simplicial complexes in a Euclidean space. Discrete Comput. Geom. 23, 293-302 (2000).

[46] R. Penrose, J. H. C. Whitehead, and E. C. Zeeman. Imbedding of manifolds in Euclidean space. Ann. Math. 73, 613-623 (1961).

[47] C. Reinhardt. Zu Möbius' Polyedertheorie. Berichte über d. Verhandl. d. Kgl. Sächs. Ges. d. Wiss., Math.-Phys. Cl. 37, 106-125 (1885).

[48] J. Richter-Gebert. Realization Spaces of Polytopes. Lecture Notes in Mathematics 1643. Springer-Verlag, Berlin, 1996.

[49] G. Ringel. Wie man die geschlossenen nichtorientierbaren Flächen in möglichst wenig Dreiecke zerlegen kann. Math. Ann. 130, 317-326 (1955).

[50] T. Rörig. Polyhedral Surfaces, Polytopes, and Projections. Dissertation. Technische Universität Berlin, 2009, 126 pages.

[51] T. Rörig and R. Sanyal. Non-projectability of polytope skeleta. In preparation.

[52] T. Rörig and G. M. Ziegler. Polyhedral surfaces in wedge products. In preparation.

[53] L. Schewe. Satisfiability Problems in Discrete Geometry. Dissertation. Technische Universität Darmstadt, 2007, 101 pages.

[54] L. Schewe. Non-realizable minimal vertex triangulations of surfaces: Showing nonrealizability using oriented matroids and satisfiability solvers. arXiv:0801.2582, 2008, 14 pages. 
[55] G. Schild. Some minimal nonembeddable complexes. Topology Appl. 53, 177-185 (1993).

[56] J. Simutis. Geometric Realizations of Toroidal Maps (Ph.D. Thesis). University of California, Davis, 1977.

[57] E. Steinitz. Polyeder und Raumeinteilungen. Encyklopädie der mathematischen Wissenschaften mit Einschluss ihrer Anwendungen, Dritter Band: Geometrie, III.1.2., Heft 9 (W. Fr. Meyer and H. Mohrmann, eds.), Chapter III A B 12, 1-139. B. G. Teubner, Leipzig, 1922.

[58] E. Steinitz and H. Rademacher. Vorlesungen über die Theorie der Polyeder unter Einschluß der Elemente der Topologie. Grundlehren der mathematischen Wissenschaften 41. Springer-Verlag, Berlin, 1934. Reprint, 1976.

[59] A. Stuart and J. K. Ord. Kendall's Advanced Theory of Statistics. Volume 2: Classical Inference and Relationships. Edward Arnold, London, 1987.

[60] T. Sulanke. Generating irreducible triangulations of surfaces. arXiv:math.C0/0606687, 2006, 11 pages.

[61] T. Sulanke. Irreducible triangulations of low genus surfaces. arXiv:math.C0/0606690, 2006, 10 pages.

[62] T. Sulanke. Source for surftri and lists of irreducible triangulations. http://hep. physics.indiana.edu/ tsulanke/graphs/surftri/, 2005. Version 0.96.

[63] T. Sulanke and F. H. Lutz. Isomorphism free lexicographic enumeration of triangulated surfaces and 3-manifolds. arXiv:math.C0/0610022v3, 2007, 24 pages; Eur. J. Comb., to appear.

[64] L. Szilassi. Les toroïdes réguliers/Regular toroids. Topologie Struct. 13, 69-80 (1986).

[65] D. Timmreck. Necessary conditions for geometric realizability of simplicial complexes. Discrete Differential Geometry (A. I. Bobenko, P. Schröder, J. M. Sullivan, and G. M. Ziegler, eds.). Oberwolfach Seminars 38, 215-233. Birkhäuser, Basel, 2008.

[66] W. T. Tutte. How to draw a graph. Proc. Lond. Math. Soc., III. Ser. 13, 743-768 (1963).

[67] E. R. van Kampen. Komplexe in euklidischen Räumen. Abh. Math. Sem. Univ. Hamburg 9, 72-78 (1932). Berichtigungen dazu: ibid 152-153.

[68] H. Whitney. The self-intersections of a smooth $n$-manifold in $2 n$-space. Ann. Math. 45, 220-246 (1944).

[69] G. M. Ziegler. Lectures on Polytopes. Graduate Texts in Mathematics 152. SpringerVerlag, New York, NY, 1995. Revised edition, 1998.

[70] G. M. Ziegler. Nonrational configurations, polytopes, and surfaces. Math. Intell. 30, No. 3, 36-42 (2008). 
[71] G. M. Ziegler. Polyhedral surfaces of high genus. Discrete Differential Geometry (A. I. Bobenko, P. Schröder, J. M. Sullivan, and G. M. Ziegler, eds.). Oberwolfach Seminars 38, 191-213. Birkhäuser, Basel, 2008.

Stefan Hougardy

Universität Bonn

Forschungsinstitut für Diskrete Mathematik

Lennéstr. 2

53113 Bonn

Germany

hougardy@or .uni-bonn.de

Frank H. Lutz

Technische Universität Berlin

Institut für Mathematik

Straße des 17. Juni 136

10623 Berlin

Germany

lutz@math.tu-berlin.de

Mariano Zelke

Humboldt-Universität zu Berlin

Institut für Informatik

Unter den Linden 6

10099 Berlin

Germany

zelke@informatik.hu-berlin.de 